\title{
Houston, we have a problem ... lapsus révélateur!
}

Ariane Treu, Georges Gabris, Maurice Hurni, Nick Miller, Lito Panayotopoulos, Alain Porchet

Comité du Groupement des psychiatres-psychothérapeutes membres de la Société Vaudoise de Médecine

1 Herren D. Houston, we have a problem ... Bull Méd Suisses. 2009;90(37):1405

2 Porter ME, Olmsted Teisberg E. Redefining Health Care, creating Value-Based Competition on Results. Harvard Business School Publishing. May 2006.

3 Gori R, Del Volgo MJ. Exilés de l'intime, la médecine et la psychiatrie au service du nouvel ordre économique. Denoël; 2008. Ainsi que: La santé totalitaire. Denoël 2005

Correspondance: Dr Ariane Treu

Vice-présidente du GPPV

Rue Beau-Séjour 7

CH-1003 Lausanne

a.treu@bluewin.ch
Dans son éditorial du Bulletin des médecins suisses no. 37, daté du 9.9.2009 et intitulé «Houston, we have a problem ...» le Dr Daniel Herren, membre du comité central de la FMH, évoque l'atterrissage sur la lune en juillet 1969 comme métaphore illustrant la réalisation d'un objectif commun dans lequel les intérêts collectifs ont primé sur les velléités individuelles. Il souhaite que la politique de santé s'en inspire pour un projet d'envergure.

Mais quel projet d'envergure nous propose-t-il? «Un modèle de concurrence basée sur la valeur, valuebased competition (...) ce qui équivaut presque à un premier pas sur la lune»[1]. Selon les deux économistes ultralibéraux qui défendent dans leur livre [2] 1'application de ce concept au système de santé, «valuebased competition is the type of competition we see in virtually every field: retailing, airlines, financial services, aerospace and computer services». Ainsi c'est un concept économique, applicable à toutes les industries, sous-tendu par le mythe d'une régulation naturelle du marché, qui devrait être le nouvel idéal de la politique de santé. Mais cette concurrence basée sur la valeur a besoin d'informations permettant de définir la valeur à l'aide d'échelles, de mesures d'évaluation. Les principes de la «value-based competition» sont les suivants: concurrence basée sur les résultats, une haute qualité des soins devrait être moins chère, la concurrence ne doit pas être locale mais nationale ...

Cette concurrence basée sur la valeur nécessitera transparence et qualité, évaluation généralisée, normes, traçabilité, conformité à des procédures standardisées pour fournir les informations sur lesquelles reposera le nouvel ordre économico-médical censé garantir une meilleure médecine où tout le monde serait gagnant. Dans cette nouvelle religion du chiffre, de la statistique et des procédures administratives de conformité la forme prend le pas sur le fond. On voit bien l'enjeu que représente dans ce contexte la manière dont on va définir la qualité et comment on va la mesurer pour la transformer en marchandise afin de faire entrer la médecine dans le paradis du business et des entreprises qui nous promet la lune.

Nous ne partageons pas ce point de vue.

Il ne s'agit pas de nier la nécessité d'un projet d'envergure qui puisse mobiliser les intérêts collectifs des médecins et des citoyens pour améliorer encore notre système de soins. Mais ce projet doit, selon nous, être pensé en termes de réalité politique et humaine, en termes de responsabilité et de subjectivité. Quelles valeurs, quel fondement éthique défendonsnous? Depuis quand la régulation naturelle du marché produit-elle l'harmonie sociale et la qualité? Il suffit de voir la crise actuelle pour se convaincre du contraire.

Force est de constater que cette idéologie d'un modèle économique auto-régulateur imprègne déjà sournoisement les théories et les pratiques médicales qu'elles recomposent. Ainsi, les psychiatres doivent lutter au quotidien contre cette idéologie qui aimerait que leur pratique ne s'embarrasse plus des concepts de réalité psychique, d'angoisse, de culpabilité et que seules s'imposent les techniques de rentabilité comportementale, les stratégies de management des conduites. C'est pourquoi il ne s'agit pas que d'une question de vocabulaire. Les mots ne sont pas neutres, ils sont porteurs d'un système de valeurs. Lorsque l'on parle de concurrence basée sur la valeur, le référentiel sous- jacent est la logique du marché et la morale libérale utilitariste et non plus une éthique de responsabilité. Cette idéologie se présente souvent dissimulée derrière un discours de simple logique des faits, on ne pourrait pas faire autrement, la réalité serait ainsi faite etc., ce qui a un effet démobilisateur sur le citoyen qui se sent impuissant. Mais l'anonymat de ces concepts censés être objectifs masque la présence des décideurs qui orientent des choix de société en dehors du débat démocratique et du champ politique où ils devraient légitimement se situer.

Concevoir le capitalisme financier de notre époque comme un palier supplémentaire de notre progression sur l'échelle du progrès est, au mieux, une façon angélique d'être dans la lune, mais vu les enjeux il serait temps de se réveiller. D'ailleurs, le Dr Herren a fait un lapsus révélateur puisque le titre de son article concerne non pas les premiers pas d'Armstrong sur la lune en 1969 mais l'échec de la mission Apollo 13.

«Houston, we have a problem ...»: ce message de détresse fut lancé le 13 avril 1970 par l'astronaute Jim Lovell après 55 heures de vol à bord d'Apollo 13 suite à l'explosion soudaine d'un réservoir d'oxygène. Pendant 4 jours d'angoisse des millions de téléspectateurs assistèrent en direct aux efforts déployés par la NASA pour ramener les trois hommes sur Terre. Et c'est là que fut l'exploit.

L'exploit aujourd'hui serait de conjuguer tous nos efforts pour éviter au système de santé suisse cette dérive ultralibérale qui risque de désintégrer toutes les valeurs de l'éthique médicale.

Pour réfléchir à ce thème récurrent, le Groupement des psychiatres-psychothérapeutes vaudois a invité le Professeur Roland Gori, auteur de plusieurs ouvrages sur ce sujet [3], à faire une conférence publique à Lausanne, à l'Hôtel de la Paix, le vendredi 13 novembre 2009 à 20 heures. Venez nombreux et débattons-en. 